\begin{tabular}{|c|l|}
\hline Title & Water entry of a superhydrophobic low-density sphere \\
\hline Author(s) & Ueda, Y.; Tanaka, M.; Uemura, T.; Iguchi, M. \\
\hline Citation & Journal of Visualization, 13(4), 289-292 \\
https://doi.org/10.1007/312650-010-0054-x \\
\hline Issue Date & 2010-11 \\
\hline Doc URL & http://hdl.handle.net/2115/47348 \\
\hline Rights & The original publication is available at www.springerlink.com \\
\hline Type & article (author version) \\
\hline File Information & JV134_289-292.pdf \\
\hline
\end{tabular}

Instructions for use 


\title{
Water entry of a superhydrophobic low-density sphere
}

\author{
Y. Ueda · M. Tanaka - T. Uemura · M. \\ Iguchi
}

the date of receipt and acceptance should be inserted later

\begin{abstract}
This paper virtually demonstrates an air cavity formed by a superhydrophobic low-density sphere entering into water with the use of a high-speed camera. Unlike the previous results for a high-density sphere, the low-density sphere pinches off the resulting air cavity at the shorter depth before the cavity has fully grown, and attracts minimal air on the rear surface due to rapid deceleration of the sphere in water. The analytical model previously proposed on the time evolution of the cavity shape is thus shown to be no longer valid for the low-density sphere.
\end{abstract}

Keywords Air cavity · Hydrophobic surface · Entry into water · Impact · Low-density sphere

\section{Introduction}

In a steelmaking process, the micro particles such as calcium oxide $(\mathrm{CaO}$, desulphurization chemical) should be effectively injected into a molten iron bath (solid-liquid impact) and dispersed in the whole bath to enhance the efficiency of desulphurization or dephosphorization. However, the injected particles attract gas bubbles due to the poor wettability with molten iron so that the dispersion in the bath could be inhibited. To achieve the effective dispersion of the injected particles, we need to investigate the dynamic behavior of the air cavity formed by the liquid entry of a particle.

Pioneering work of solid-liquid impact can be found in Worthington \& Cole (1897, 1900) who used single-spark photography to examine the air cavity formed by the vertical entry of a sphere into water. Recently, with the progress of a high-speed camera, several kinds of articles virtually investigate the air cavity formed by a superhydrophobic high-density sphere entering into water (see Duez et al., 2007) together with mathematical formulation within an inviscid framework (see Aristoff \& Bush, 2009; Duclaux et al., 2007; Lee \& Kim. 2008; Yan et al., 2009). This paper virtually demonstrates

Y. Ueda · M. Tanaka · M. Iguchi

Division of Materials Science and Engineering, Hokkaido University, Hokkaido 060-8628, Japan

E-mail: y-ueda@eng.hokudai.ac.jp

T. Uemura

Department of Mechanical Engineering, Kansai University, Osaka 564-8680, Japan 
the water entry of a superhydrophobic lower density sphere than water and adopts the previous analytical model to the low-density sphere on the time evolution of the cavity shape by Duclaux et al. (2007) and Aristoff \& Bush (2009).

\section{Experimental setup and visualization procedure}

The growth and pinch-off of the air cavity due to the entry of a superhydrophobic low-density sphere into water is demonstrated experimentally. The low-density sphere, having a density $\rho_{p}=851$ or $920 \mathrm{~kg} / \mathrm{m}^{3}$ and a diameter $d_{p}=12.7 \mathrm{~mm}$, is freely released, by putting off the power supply of the vacuum pump, at a certain height $(40 \leq h \leq 200 \mathrm{~mm})$ from a static water surface level in a transparent acrylic cubic vessel $(300 \mathrm{~mm}$ each). After freely released, the sphere impacts on the water surface and forms the air cavity behind it. The sphere presently used is a lower density than the water so that the sphere naturally broaches from a certain depth of the dive. Several kinds of the cavities formed by the entry of the dewetting sphere are recorded with a high-speed camera whose frame rate is 500 frames/s with the shutter speed of $250 \mu \mathrm{s}$ for the resolution $1024 \times 1260$ pixels.

\section{Results and discussion}

A coating of water-repellent material dewets a solid surface. Indeed, an air-layer is observed to cover the surface of a hydrophobic sphere immersed in water (see Tanaka et al., 2008a). As shown in Aristoff \& Bush (2009), Duez et al. (2007) and Tanaka et al. (2008b), the entry of the hydrophobic sphere into water is known to form the air cavity whereas the non-coating (smooth surface) sphere seems not to form it. The resulting air cavity can be observed at the pinch-off event in a various kinds of patterns which are dependent on a released height of the sphere (see the top side of Figs. 1 and 2 ), i.e., the distinct ripple can be observed on the cavity surface when the sphere is released at the higher position than $h \geq 120 \mathrm{~mm}$. Figure 4 shows a video sequence of the water entry of the hydrophobic sphere from the onset of the impact. Unlike the previous experiments adopting the high-density sphere (see e.g., Figs. 7-8 of Duclaux et al. (2007) or Figs. 5-7 of Aristoff \& Bush (2009)), the low-density sphere pinches off the air cavity at the shorter depth and attracts minimal air on the rear surface because of rapid deceleration of the sphere in water (see the last of Fig. 4 and compare the pinch-off point between Fig. 3 and the top side of Figs. 1 and 2). According to Duclaux et al. (2007) and Aristoff \& Bush (2009), the water entry of the high-density sphere would form the cavity surface from around the separation point due to a pressure drop at the impact. In contrast, the low-density sphere experiences a lower pressure drop which could result in a closure of the air cavity before the cavity has fully grown (i.e., a growing velocity of the cavity in the radial direction could be weak).

As mentioned in the previous section, the lower density sphere than water is presently used so that the sphere can experience the rapid deceleration (after then, it broaches from a certain depth of the dive). The equation of motion of a hydrophobic sphere entering into water was derived by Lee \& Kim (2008) and briefly presented in Aristoff \& Bush (2009). Instead of solving the equation of motion to which the solution cannot be analytically obtained, we measure the instantaneous position of the 

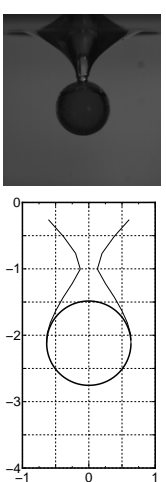

$h=40 \mathrm{~mm}$
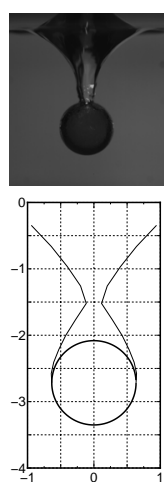

$h=60 \mathrm{~mm}$
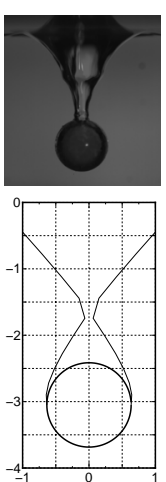

$h=80 \mathrm{~mm}$
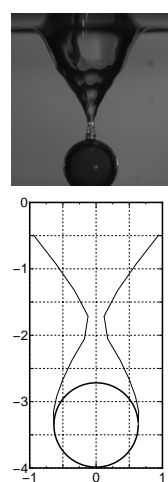

$h=120 \mathrm{~mm}$
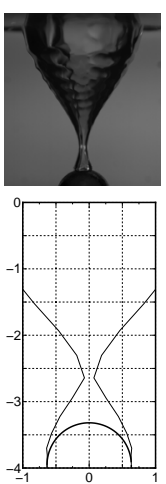

$h=200 \mathrm{~mm}$

Fig. 1 Air cavity shapes, at pinched off, formed by the water entry of a superhydrophobic low-density sphere $\left(d_{p}=12.7 \mathrm{~mm}\right.$ and $\left.\rho_{p}=851 \mathrm{~kg} / \mathrm{m}^{3}\right)$. Top photograph, Bottom analytical model of Duclaux et al. (2007).
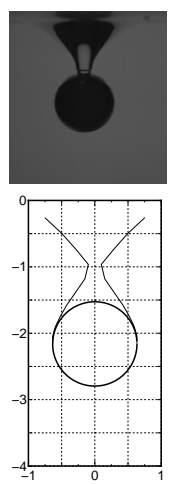

$h=40 \mathrm{~mm}$
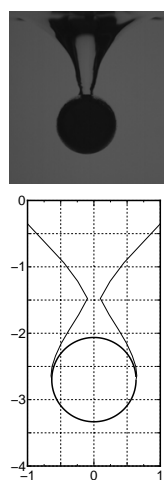

$h=60 \mathrm{~mm}$
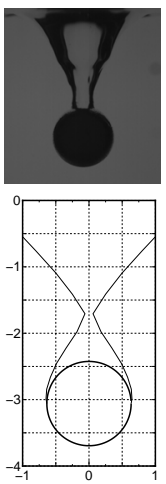

$h=80 \mathrm{~mm}$
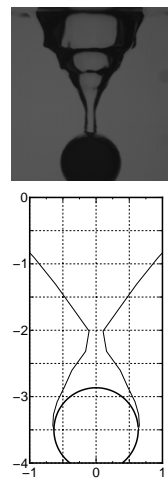

$h=120 \mathrm{~mm}$
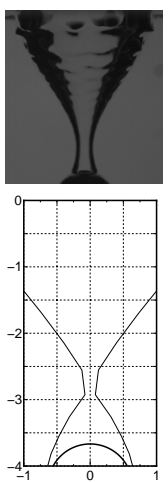

$h=200 \mathrm{~mm}$

Fig. 2 Air cavity shapes, at pinched off, formed by the water entry of a superhydrophobic low-density sphere $\left(d_{p}=12.7 \mathrm{~mm}\right.$ and $\left.\rho_{p}=920 \mathrm{~kg} / \mathrm{m}^{3}\right)$. Top photograph, Bottom analytical model of Duclaux et al. (2007).

sphere entering into water with the high-speed camera (see Fig. 5). The entering velocity of the sphere is calculated from Fig. 5. As observed in Fig. 5, the low-density sphere experiences the rapid deceleration, whereas the high-density sphere sinks with the constant velocity in water (see Duclaux et al., 2007; and Aristoff \& Bush, 2009). Duclaux et al. (2007) and Aristoff \& Bush (2009), who extended the work of Duclaux et al. (2007) by including the effects of surface tension and aerodynamic pressure on the cavity surface, analytically obtained the evolution of the cavity shape on the basis of the Besant-Rayleigh problem by assuming the diameter of the cavity to be small relative to its length (i.e., the cavity shape is slender, which allows us to treat in $2 \mathrm{D}$ ). The time evolution of the cylindrical cavity radius $R$ at a given $z$ is approximately described as $R^{2}=\left(d_{p} / 2\right)^{2}+\sqrt{\alpha} v_{p} d_{p} t-g z t^{2}$, where we have used the initial conditions $R(t=0)=d_{p} / 2$ and $\dot{R}(t=0)=\sqrt{\alpha} v_{p 0}$ with $\alpha$ a constant smaller than unity (see Duclaux et al., 2007). In addition, $v_{p 0}$ is the entering velocity of a sphere into water $\left(v_{p 0}=\sqrt{2 g h}\right)$ and $g$ is acceleration due to gravity. The calculated shapes of the cavity are compared to the experimental photograph in Figs. 1 and 2. It seems that the above 


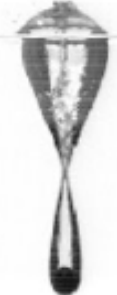

Fig. 3 Cavity due to a high-density sphere $\left(\rho_{p}=\right.$ $2360 \mathrm{~kg} / \mathrm{m}^{3}$ ) is cited from Fig.7 of Duclaux et al. (2007).

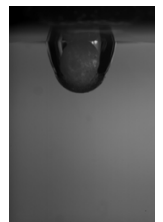

$t=8.0 \mathrm{~ms}$

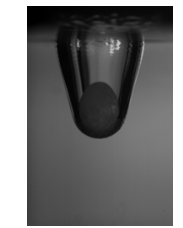

$t=16.0 \mathrm{~ms}$

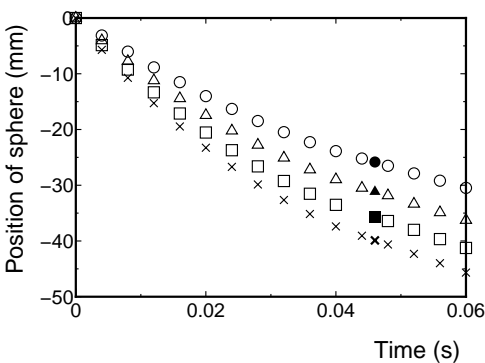

Fig. 5 Position of a sphere $\left(d_{p}=12.7 \mathrm{~mm}\right.$ and $\rho_{p}=$ $851 \mathrm{~kg} / \mathrm{m}^{3}$ ) entering into water. Circle: $h=50 \mathrm{~mm}$ Triangle: $h=80 \mathrm{~mm}$, Square: $h=120 \mathrm{~mm}$, Cross: $h=160 \mathrm{~mm}$. Data at the pinch-off event is indicated as solid/bold symbol.

Fig. 4 A video sequence of the water entry of a hydrophobic sphere from the onset of the impact to the pinch-off in the case of $d_{p}=12.7 \mathrm{~mm}, d_{p}=851 \mathrm{~kg} / \mathrm{m}^{3}$ and $h=160 \mathrm{~mm}$.

equation is no longer valid in the case of the pinch-off position being close to the sphere (i.e., Although Duclaux et al. (2007) and Aristoff \& Bush (2009) do not restrict the formulations to a high-density sphere, a low-density sphere pinches off the cavity before the cavity has fully grown, which fails to fulfill the above-mentioned assumption of the cavity shape being slender). To remedy this, the advanced analysis will be required in a three-dimensional problem. We intend to analyze the problem in a future work.

\section{References}

1. Aristoff, J.M. and Bush, J.W.M., Water entry of small hydrophobic spheres, J. Fluid Mech., 619, 45-78, (2009).

2. Duclaux, V., Caillé, F., Duez, C., Ybert, C., Bocquet, L. and Clanet, C., Dynamics of transient cavities, J. Fluid Mech., 591, 1-19, (2007).

3. Duez, C., Ybert, C., Clanet, C. and Bocquet, L., Making a splash with water repellency, Nature Physics, 3, 180-183, (2007).

4. Lee, D.-G. and Kim, H.-Y., Impact of a superhydrophobic sphere onto water, Langmuir, 24, 142-145, (2008).

5. Tanaka, M., Hashimoto, M, Tsujino, R. and Iguchi, M., Model experiment on the hydrodynamic drag of a poorly wetted sphere penetrating into a molten metal bath, J. JSEM, 8-3, 196-200, (2008a).

6. Tanaka, M., Hashimoto, M, Tsujino, R. and Iguchi, M., Behavior of a poorly wetted lowdensity sphere penetrating into a water bath (in Japanese), J. JSEM, 8-3, 201-205, (2008b).

7. Worthington, A.M. and Cole, R.S., Impact with a liquid surface, Studied by the aid of instantaneous photography, Phil. R. Soc. London, A 189, 137-148, (1897).

8. Worthington, A.M. and Cole, R.S., Impact with a liquid surface studied by the aid of instantaneous photography. Paper II, Phil. R. Soc. London, A 194, 175-199, (1900).

9. Yan, H., Liu, Y., Kominiarczuk, J. and Yue, D.K.P., Cavity dynamics in water entry at low Froude numbers, J. Fluid Mech., 641, 441-461, (2009). 